\title{
Axial Force at the Vessel Bottom Induced by Axial Impellers
}

\author{
I. Fořt, P. Hasal, A. Paglianti, F. Magelli
}

\begin{abstract}
This paper deals with the axial force affecting the flat bottom of a cylindrical stirred vessel. The vessel is equipped with four radial baffles and is stirred with a four $45^{\circ}$ pitched blade impeller pumping downwards. The set of pressure transducers is located along the whole radius of the flat bottom between two radial baffles. The radial distribution of the dynamic pressures indicated by the transducers is measured in dependence on the impeller off-bottom clearance and impeller speed.

It follows from the results of the experiments that under a turbulent regime of flow of an agitated liquid the mean time values of the dynamic pressures affecting the bottom depend not on the impeller speed but on the impeller off-bottom clearance. According to the model of the flow pattern of an agitated liquid along the flat bottom of a mixing vessel with a pitched blade impeller, three subregions can be considered in this region: the liquid jet streaming downwards from the impeller deviates from its vertical (axial) direction to the horizontal direction, the subregion of the liquid flowing horizontally along the bottom and, finally, the subregion of the liquid changing direction from the bottom upwards (vertically) along the wall of the cylindrical vessel, when the volumetric flow rates of the liquid taking place in the downward and upward flows are the same.
\end{abstract}

Keywords: pitched blade impeller, dynamic pressure, mixing vessel, flat bottom, pressure transducer.

\section{Introduction}

Axial high-speed rotary impellers (propeller impellers, pitched blade impellers, axial hydrofoil impellers) create a significant axial force (thrust). The rotation of the impeller causes a field of axial forces by which the flowing liquid acts on the impeller and the vessel. It is relatively easy to determine such forces and their components, and it can be done without interfering with the velocity field. Until now, the axial force of the axial flow impellers has been determined directly as the change in vessel weight [1] or from the distribution of the axial force affecting the bottom [2-4]. From the latter investigation, it follows that the distribution of the axial force affecting the flat bottom of the cylindrical mixing vessel is unambiguously joined with the flow pattern along the bottom: Force $f_{\mathrm{ax} 1}$ originates when the liquid jet streaming downwards from the impeller rotor region deviates from its vertical (axial) direction and flows along the vessel bottom, and force $f_{\text {ax2 }}$ appears in the region where the liquid flowing along the vessel bottom changes direction and starts flowing vertically (axially) up the cylindrical vessel walls. Between the areas of the bottom affected by forces $f_{\mathrm{ax} 1}$ and $f_{\mathrm{ax} 2}$ there is, under certain conditions (e.g. impeller off-bottom clearance), a subregion where the pressure force acts on this corresponding area.

In experiments, most attention has been paid to the distribution of the axial pressures along the bottom. These distributions were determined directly $[2,3]$ by measuring the total pressures in holes situated in the bottom of the vessel. The dynamic pressures corresponding to them were calculated as the differences between the total pressure measured and the hydrostatic pressure under the conditions of the experiment. Direct measurement of the dynamic pressures affecting the wall of the mixing vessel $[5,6]$ has recently been developed. This approach consists of a non-invasive measurement by means of pressure transducers located in appropriate positions of the boundary of the stirred system (wall, bottom), simultaneously recording the measured instantaneous signal throughout the experiment. Such an experimental technique allows us to determine not only the mean value of the measured quantity but also some characteristics of the oscillations.

The aim of this study is to determine the distribution of the dynamic pressures along the flat bottom of a pilot plant cylindrical mixing vessel equipped with four radial baffles and stirred with a four $45^{\circ}$ pitched blade impeller pumping downwards. A set of pressure transducers is located along the whole radius of the flat bottom between two adjacent baffles. The radial distribution of the dynamic pressures indicated by the pressure transducers will be determined in dependence on the impeller off-bottom clearance and the impeller speed. The results of the experiments are evaluated according to the model idea of the flow along the flat bottom in a cylindrical mixing vessel with an axial high-speed impeller under turbulent regime of flow of an agitated liquid.

\section{Experimental}

The experiments were performed in a flat-bottomed cylindrical stirred vessel of inner diameter $T=0.49 \mathrm{~m}$ filled with water at room temperature with the vessel height $H=T$. The

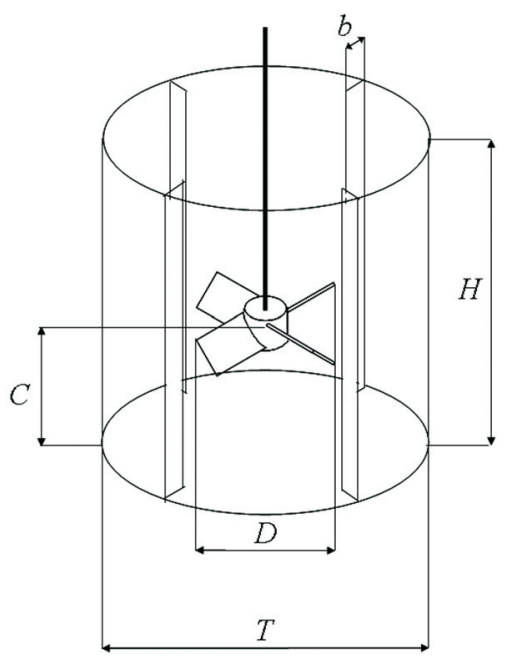

Fig. 1: Pilot plant experimental equipment 


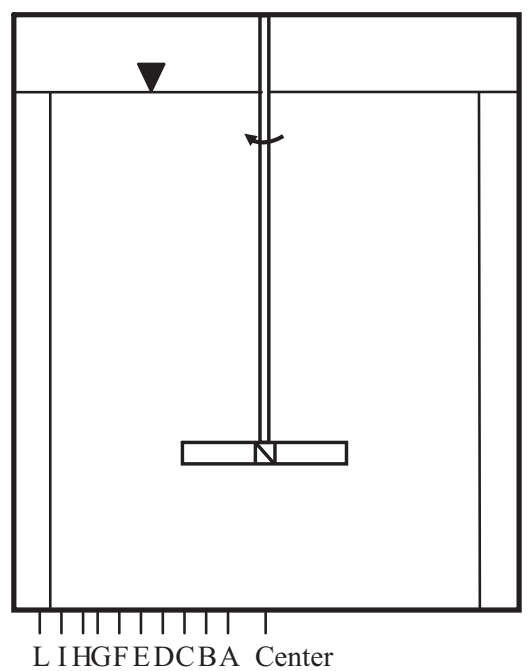

\begin{tabular}{|c|c|c|c|c|c|c|c|c|c|c|}
\hline Distance & Distance & Distance & Distance & Distance & Distance & Distance & Distance & Distance & Distance & Distance \\
I Wall & $\mathrm{IL}$ & $\mathrm{HI}$ & $\mathrm{GH}$ & $\mathrm{FG}$ & $\mathrm{EF}$ & $\mathrm{DE}$ & $\mathrm{CD}$ & $\mathrm{BC}$ & $\mathrm{A}-\mathrm{B}$ & $\mathrm{A}$ Center \\
$\mathrm{cm}$ & $\mathrm{cm}$ & $\mathrm{cm}$ & $\mathrm{cm}$ & $\mathrm{cm}$ & $\mathrm{cm}$ & $\mathrm{cm}$ & $\mathrm{cm}$ & $\mathrm{cm}$ & $\mathrm{cm}$ & $\mathrm{cm}$ \\
3 & 1.8 & 2 & 1.5 & 2.2 & 1.8 & 2 & 2 & 2 & 2.2 & 4 \\
\hline
\end{tabular}

Fig. 2: Distribution of pressure transducers along the bottom

vessel was equipped with four radial baffles (width of baffles $b=0.1 T$ ) and was stirred with a four pitched blade impeller (pitch angle $\alpha=45^{\circ}$, diameter $D=(2 / 5) T$, width of blade $W=D / 5$ ), pumping downward. Two levels of impeller off-bottom clearances $C=T / 3, T / 4$ and two levels of impeller speed $n=284 \mathrm{rpm}$ and $412 \mathrm{rpm}$ were adjusted (see Fig. 1).

In this work, the total pressure time series were recorded using a set of nine pressure transducers distributed along the flat bottom between two adjacent baffles (see Fig. 2). The transducer is based on a silica chip and a slim diaphragm $2.54 \mathrm{~mm}^{2}$ in surface area, which is able to reveal small pressure variations. The time traces were recorded and transferred to a personal computer by a data acquisition system card, and were controlled by a simple LabView programme. For each experimental run, a time history of about 60 minutes was recorded at a sampling frequency of $500 \mathrm{~Hz}$.

\section{Results of experiments}

The mean time values of the local total axial pressures detected by the pressure transducers were calculated from the measured total pressure time series. Then the mean time values of the local dynamic axial pressures $p_{\mathrm{ax}}$ were calculated as the difference between the local mean total axial pressure and the hydraulic pressure given by the height of the liquid level $H$. The calculated results from each position of the pressure transducer were the mean time value of the local dynamic pressure $\overline{p_{\mathrm{ax}}}$ and its standard deviation $s_{p_{\mathrm{sx}}}$, always at the given (selected) value of the off-bottom clearance and the impeller speed. Dimensional quantity $\overline{p_{\mathrm{ax}}}$ was expressed in dimensionless form

$$
P_{\mathrm{ax}}=\frac{\overline{p_{\mathrm{ax}}}}{\rho n^{2} D^{2}},
$$

and plotted in dependence on the dimensionless radial coordinate $R$, where quantity $r$ is a radial coordinate with its origin in the centre of the circular bottom:

$$
R=\frac{2 r}{T} \text {. }
$$

Figs. 3 and 4 illustrate the above mentioned radial profiles of the mean time local dynamic pressure, always at two levels of impeller speed.

A statistical analysis of the fluctuating local dynamic pressures resulted in calculating the standard deviation of this quantity $s_{p_{\mathrm{sx}}}$. These statistical characteristics were expressed in dimensionless form

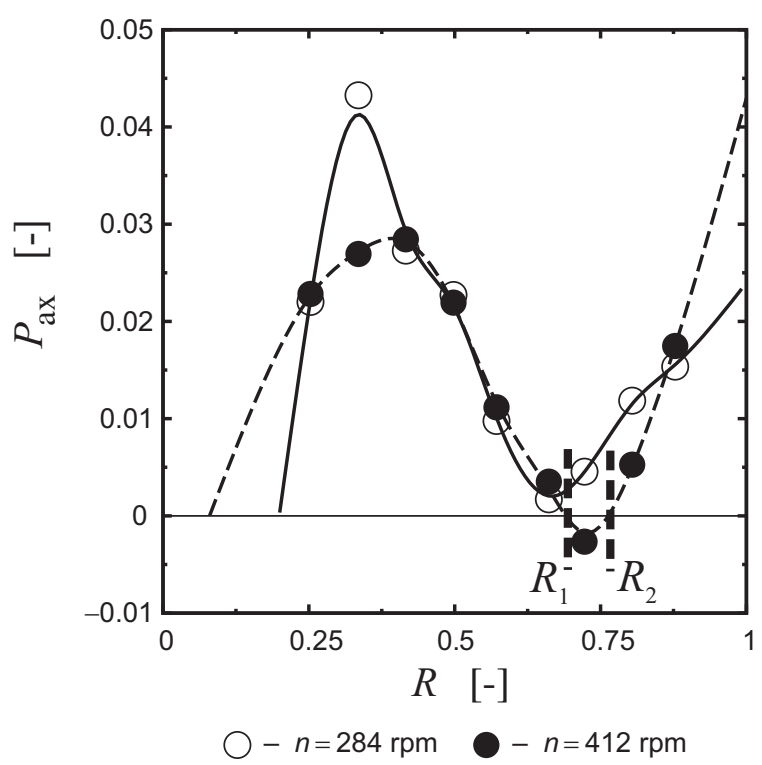

Fig. 3: Radial profiles of the dimensionless mean time local axial dynamic pressures on the vessel bottom $(\mathrm{C}=\mathrm{T} / 3)$ 


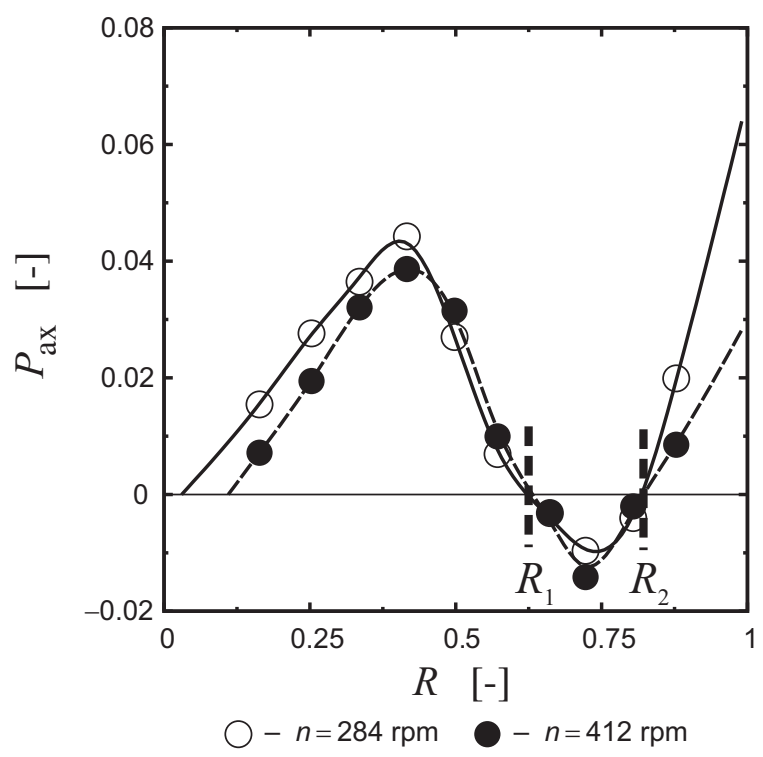

Fig. 4: Radial profiles of the dimensionless mean time local axial dynamic pressures on the vessel bottom $(\mathrm{C}=\mathrm{T} / 4)$

$$
S_{p_{\mathrm{ax}}}=\frac{s_{p_{\mathrm{ax}}}}{\rho n^{2} D^{2}},
$$

or as the variance

$$
V_{p_{\mathrm{ax}}}=\frac{s p_{\mathrm{ax}}}{\overline{p_{\mathrm{ax}}}}
$$

Figs. 5 and 6 illustrate the radial profiles of quantity $S_{p_{\mathrm{sx}}}$, and Figs. 7 and 8 illustrate the radial profiles of quantity $V_{p_{\mathrm{sx}}}$, always in dependence on the dimensionless radial coordinate $R$.

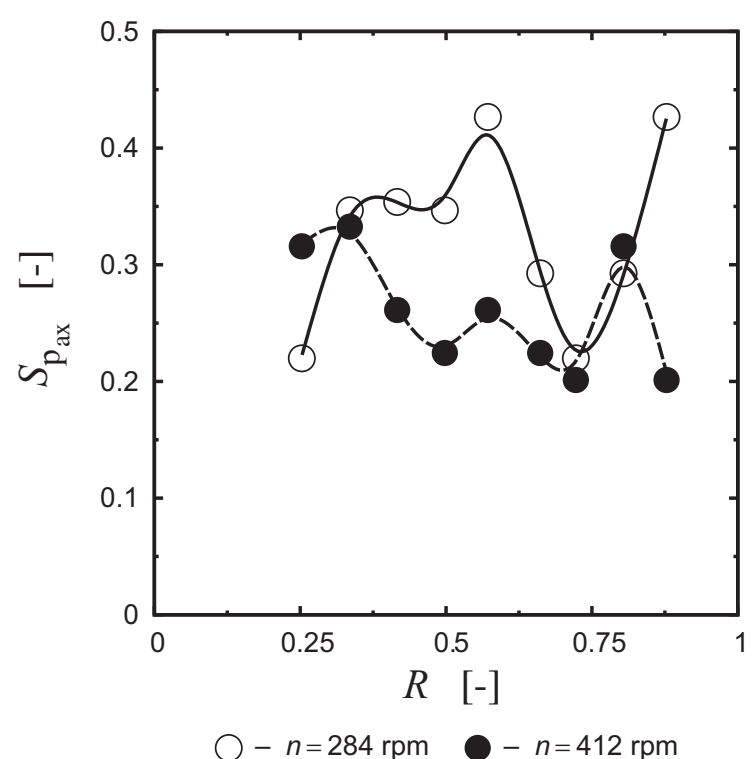

Fig. 5: Radial profiles of dimensionless standard deviations of the local axial dynamic pressures on the vessel bottom $(C=T / 3)$

In accordance with the results of previous investigations $[3,4]$ three subregions of the forces acting on the flat bottom of mixing vessel can be considered:

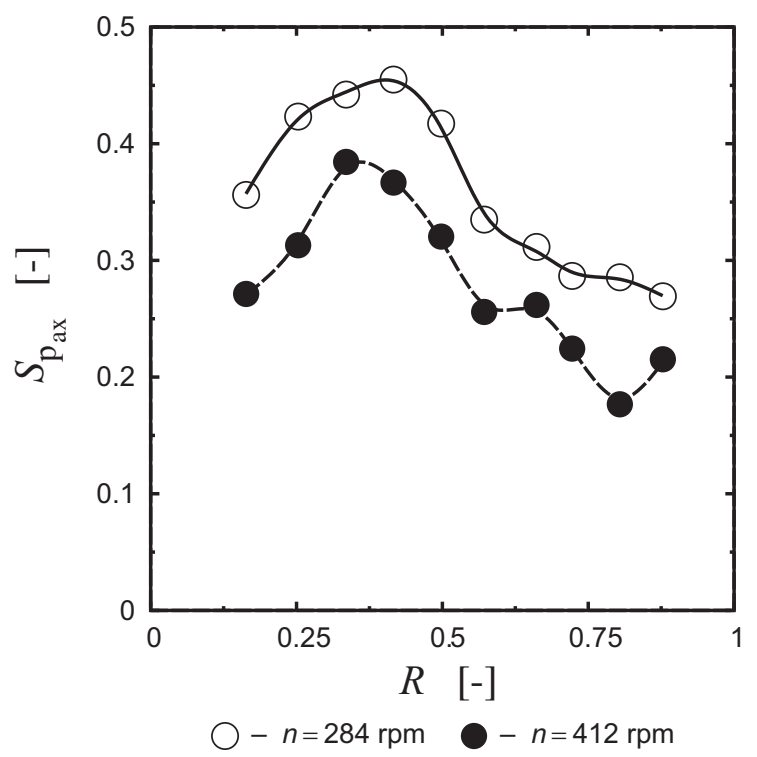

Fig. 6: Radial profiles of dimensionless standard deviations of the local axial dynamic pressures on the vessel bottom $(C=T / 4)$

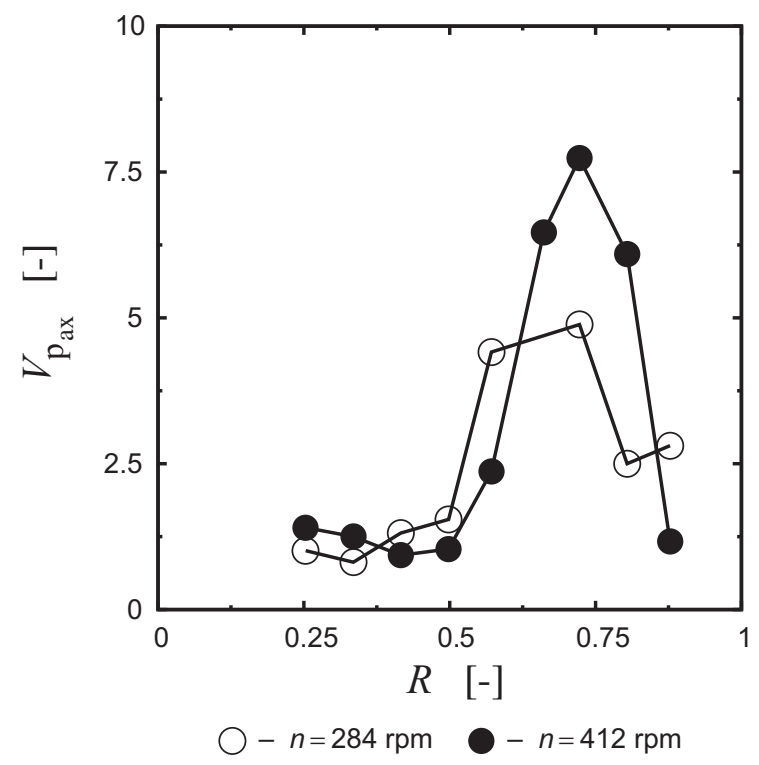

Fig. 7: Radial profiles of variances of the local axial dynamic pressures on the vessel bottom $(C=T / 3)$

1. The subregion below the axial impeller, where the liquid jet streaming downward from the impeller rotor region deviates from its direction and flows along the vessel bottom. The outer boundary of this region $R_{1}$ is given by the first intersection of the curve $P_{\mathrm{ax}}=P_{\mathrm{ax}}(R)$ and the coordinate $P_{\mathrm{ax}}=O$.

2. The subregion at the vessel wall where the liquid flowing along the vessel bottom changes direction and starts flowing up the cylindrical vessel walls. The inner boundary of its region $R_{2}$ is given by the second intersection of the curve $P_{\mathrm{ax}}=P_{\mathrm{ax}}(R)$ and the coordinate $P_{\mathrm{ax}}=O$. The outer boundary of this subregion is a radial coordinate of the vessel wall.

3. The subregion at the flat bottom where the agitated liquid flows along the bottom. Its boundaries are given by the 


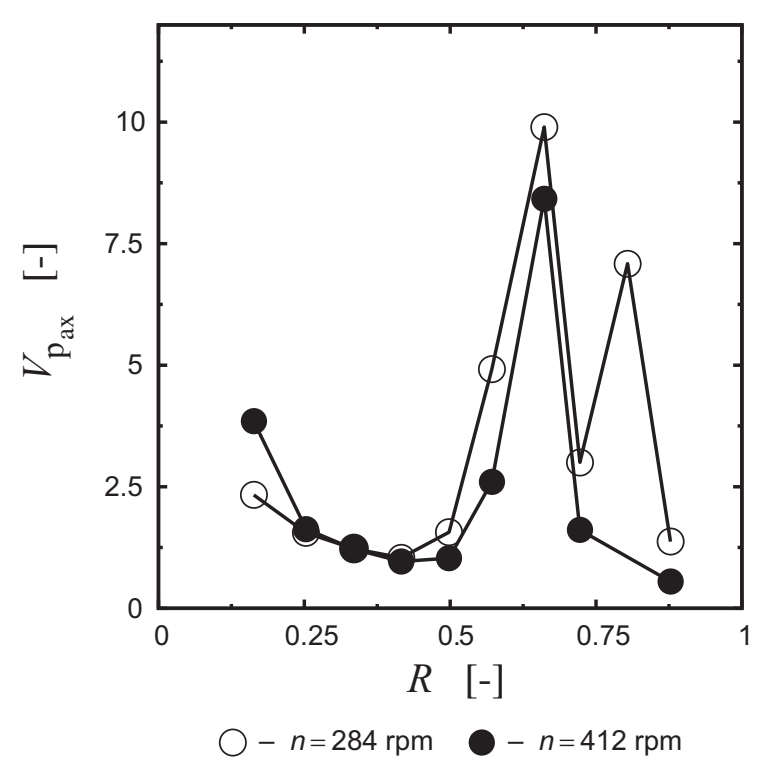

Fig. 8: Radial profiles of variances of the local axial dynamic pressures on the vessel bottom $(\mathrm{C}=\mathrm{T} / 4)$

two above mentioned intersections, $R_{1}$ (the inner one) and $R_{2}$ (the outer one).

It follows from the plots $P_{\mathrm{ax}}=P_{\mathrm{ax}}(R)$ shown in Figs. 3 and 4 , that the series of above defined subregions depend on the impeller off-bottom clearance. The higher the quantity $C / T$, the smaller the area of the second subregion, and finally, this subregion can disappear, so it holds

$$
R_{1 \max }=R_{2}=\sqrt{2} / 4 \text {. }
$$

Then the maximum value of coordinate $R_{1}$ coincides with the minimum value of coordinate $R_{2}$, corresponding to the geometrical mean of the dimensional radial coordinate $\sqrt{2} / 4$.

The mean time axial forces affecting individual subregions of the bottom can be calculated under assumption of axial symmetry of the investigated region by integrating of the mean time local dynamic pressures over the corresponding area of the bottom:

$$
\overline{f_{\mathrm{ax} 1}}=2 \pi \int_{0}^{r 1} \overline{p_{\mathrm{ax}}}(r) r \mathrm{~d} r
$$

and

$$
\overline{f_{\mathrm{ax} 2}}=2 \pi \int_{r 2}^{T / 2} \overline{p_{\mathrm{ax}}}(r) r \mathrm{~d} r .
$$

The mean time axial forces can be expressed in dimensionless form as follows

$$
F_{\text {ax } i}=\frac{\overline{f_{\text {ax } i}}}{\rho n^{2} D^{4}}, i=1,2 .
$$

Tables 1 and 2 consist of values of the calculated mean time axial forces both in dimensional form and in dimensionless form, as well as the values of boundary coordinate $R_{1}$ for both two investigated impeller off-bottom clearances. It should be pointed out that the curves $p_{\mathrm{ax}}=p_{\mathrm{ax}}(r)$ were extrapolated by a cubic function up to the vessel wall.

The values of components $\overline{f_{\mathrm{ax} 1}}$ and $\overline{f_{\mathrm{ax} 2}}$ are derived from an impulse theorem. The vertical (axial) component of the free liquid stream changes in both subregions where a $\pi / 2$-radian turn takes place [4]

$$
\begin{aligned}
\overline{f_{\mathrm{ax} 1}} & =\frac{\rho\left(Q_{b t 1}\right)^{2}}{\pi r_{1}^{2}}, \\
\overline{f_{\mathrm{ax} 2}} & =\frac{\rho\left(Q_{b t 2}\right)^{2}}{\pi \frac{T^{2}}{4-r_{2}^{2}}} .
\end{aligned}
$$

The volumetric flow rates along the bottom $Q_{b t 1}$ and $Q_{b t 2}$ act on the areas limited by radii $r_{1}$ or $R_{1}$ and $R_{2}$ or $T / 2$ and $r_{2}$, respectively, and create the mean axial forces $\overline{f_{\mathrm{ax} 1}}$ and $\overline{f_{\mathrm{ax}}}$, respectively. Both quantities can be expressed in dimensionless form

$$
N_{Q_{b t i}}=\frac{Q_{b t i}}{n D^{3}}, i=1,2 .
$$

Table 1: Axial forces affecting flat bottom (four $45^{\circ}$ pitched blade impeller, $C=T / 3$ )

\begin{tabular}{|c|c|c|c|c|c|c|c|c|}
\hline $\begin{array}{c}n \\
{[\mathrm{rpm}]}\end{array}$ & $\begin{array}{c}\overline{f_{\mathrm{ax} 1}} \\
{[\mathrm{~N}]}\end{array}$ & $\begin{array}{c}\overline{f_{\mathrm{ax} 2}} \\
{[\mathrm{~N}]}\end{array}$ & $\begin{array}{c}F_{\mathrm{ax} 1} \\
{[-]}\end{array}$ & $\begin{array}{c}F_{\mathrm{ax} 2} \\
{[-]}\end{array}$ & $\begin{array}{c}Q_{b t 1} \\
{\left[\mathrm{~m}^{3} \mathrm{~s}^{-1}\right]}\end{array}$ & $\begin{array}{c}Q_{b t 2} \\
{\left[\mathrm{~m}^{3} \mathrm{~s}^{-1}\right]}\end{array}$ & $\begin{array}{c}N_{Q_{b t 1}} \\
{[-]}\end{array}$ & $\begin{array}{c}N_{Q_{b t 2}} \\
{[-]}\end{array}$ \\
\hline 284 & 13.17 & 11.49 & 0.406 & 0.355 & 0.0329 & 0.0322 & 0.939 & 0.917 \\
\hline 412 & 25.41 & 26.03 & 0.373 & 0.382 & 0.0485 & 0.0485 & 0.899 & 0.952 \\
\hline \multicolumn{2}{|c|}{ Average values } & - & 0.390 & 0.369 & - & - & 0.919 & 0.935 \\
\hline
\end{tabular}

$R_{1}=0.340$

Table 2: Axial forces affecting flat bottom (four $45^{\circ}$ pitched blade impeller, $C=T / 4$ )

\begin{tabular}{|c|c|c|c|c|c|c|c|c|}
\hline $\begin{array}{c}n \\
{[\mathrm{rpm}]}\end{array}$ & $\begin{array}{c}\overline{f_{\mathrm{ax} 1}} \\
{[\mathrm{~N}]}\end{array}$ & $\begin{array}{c}\overline{f_{\mathrm{ax} 2}} \\
{[\mathrm{~N}]}\end{array}$ & $\begin{array}{c}F_{\mathrm{ax} 1} \\
{[-]}\end{array}$ & $\begin{array}{c}F_{\mathrm{ax} 2} \\
{[-]}\end{array}$ & $\begin{array}{c}Q_{b t 1} \\
{\left[\mathrm{~m}^{3} \mathrm{~s}^{-1}\right]}\end{array}$ & $\begin{array}{c}Q_{b t 2} \\
{\left[\mathrm{~m}^{3} \mathrm{~s}^{-1}\right]}\end{array}$ & $\begin{array}{c}N_{Q_{b t 1}} \\
{[-]}\end{array}$ & $\begin{array}{c}N_{Q_{b t 2}} \\
{[-]}\end{array}$ \\
\hline 284 & 14.86 & 14.67 & 0.459 & 0.453 & 0.0350 & 0.0364 & 0.997 & 1.036 \\
\hline 412 & 28.60 & 26.37 & 0.420 & 0.388 & 0.0486 & 0.0488 & 0.954 & 0.958 \\
\hline \multicolumn{2}{|l}{ Average values } & - & 0.440 & 0.403 & - & - & 0.976 & 0.997 \\
\hline
\end{tabular}

$R_{1}=0.320$ 
Tables 1 and 2 show the values of the calculated volumetric flow rates along the bottom in dimensional form and in dimensionless form.

Figs. 5 and 6 clearly indicate that the value of the dimensionless (and also of the dimensional) standard deviation of the local dynamic pressure depends significantly on the absolute level of the measured pressure signal. The higher the level of the signal observed at the lower impeller off-bottom clearance, $C / T=1 / 4$, gives mutually similar radial profiles of $S_{p_{\text {ax }}}$ at both examined impeller speeds, while at $C / T=1 / 3$ the variations of the dimensionless standard deviation $S_{p_{\text {ax }}}$ along the bottom radius seem to be quite irregular. The values of the coefficient of variation, $V_{p_{\text {ax }}}$, of the local bottom dynamic pressure (see Figs. 7 and 8) exhibit very similar trends at both off-bottom clearance values used in the experiments: at higher values of the mean time axial dynamic pressure, $\bar{p}_{\mathrm{ax}}$, which originates from more directional liquid flow within the region beneath the impeller and in the bottom region close to the vessel wall. The pressure fluctuations are insignificant in comparison with the fluctuations in the region close to the radius of the first turn of the liquid stream from the impeller rotor region (radius $R_{1}$ in Figs. 3 and 4 ) and close to the very beginning of the second turn at the vessel wall (radius $R_{2}$ in Figs. 3 and 4).

\section{Discussion}

All experiments were conducted under a turbulent regime of flow of an agitated liquid. This fact confirms that all results of experiments presented here, expressed in dimensionless form, depend not on the impeller speed but on the impeller off-bottom clearance. With respect to the statistical analysis of the experimental data, it seems that the accuracy of the experiments performed in the subregion between radius $r_{1}\left(R_{1}\right)$ and radius $r_{2}\left(R_{2}\right)$ of the flow along the bottom is worse than the accuracy in the two other subregions. The reason for this difference can be explained by the different flow mechanisms in these subregions and, thus, by different mechanisms of the origin of the measured axial dynamic pressures [4].

It follows from Figs. 3 and 4 that the subregion between radius $r_{1}\left(R_{1}\right)$ and radius $r_{2}\left(R_{2}\right)$ of the flow along the bottom practically diminishes at the level of impeller $C / T=1 / 3$. Then the value for radius $R_{1}$ (see Table 1 ) is rather close to the value for $R_{1 \max }$ (see Eq. 3). Moreover the values of the local axial dynamic pressure in both regions of the flow turn are signifi- cantly higher at the impeller off-bottom clearance $C / T=1 / 4$ than when the impeller is located at a higher axial distance from the bottom.

Tables 1 and 2 clearly illustrate the expected validity of the continuity equation of the flow around the bottom:

$$
Q_{b t 1}=Q_{b t 2}
$$

found earlier $[3,4]$ for the six $45^{\circ}$ pitched blade impeller. For the off-bottom impeller clearance $C=T / 3$ it is moreover valid

$$
\overline{f_{\mathrm{ax} 1}}=\overline{f_{\mathrm{ax} 2}}
$$

always at the given impeller speed.

Table 3 covers the same quantities as those observed in this study, but for the six $45^{\circ}$ pitched blade impeller published earlier $[3,4]$. The general dependence of all investigated quantities $\left(F_{\mathrm{ax}}, N_{b t}, R_{1}\right)$ on the impeller off-bottom clearance corresponds fairly well to the results in this study. The only, rather significant, difference is probably caused by the relation between the flow rate along the bottom $N_{Q_{b x}}$ and the flow rate number $N_{Q_{p}}$, i.e. the dimensionless impeller pumping capacity. The value of this quantity for the arrangement of the agitated system investigated here is [7]:

$$
N_{Q_{p}}=0.753 \text { (four } 45^{\circ} \text { pitched blade impeller). }
$$

While for the six $45^{\circ}$ pitched blade impeller the quantity $N_{Q_{b t}}$ does not amount to its pumping capacity $N_{Q_{p}}$, for the four $45^{\circ}$ pitched blade impeller it significantly exceeds this quantity. These facts are joined with different values of the pumping area at the bottom (up to radius $R_{1}$ ) which is greater for the latter impeller than for the former impeller. The direction of the flow pattern is therefore more axial for the four blade impeller than for the six blade impeller, and more induced flow is entrained from the surroundings of the impeller rotor region towards the bottom.

\section{Conclusions}

Under a turbulent regime of flow of an agitated liquid the mean time values of the dimensionless dynamic pressures affecting the bottom depend not on the impeller speed but on the impeller off-bottom clearance. According to the experimentally confirmed model of the flow pattern of an agitated liquid along the flat bottom of a mixing vessel with a pitched blade impeller, three subregions can be considered in this region: the liquid jet streaming downwards from the impeller rotor region deviates from its vertical (axial) direction to the

Table 3: Axial forces affecting flat bottom $-D / T=2 / 5$ (six $45^{\circ}$ pitched blade impeller)

\begin{tabular}{|c|c|c|c|c|}
\hline $\begin{array}{c}C / T \\
{[-]}\end{array}$ & $\begin{array}{c}F_{\mathrm{ax}} \\
{[-]}\end{array}$ & $\begin{array}{c}N_{b t} \\
{[-]}\end{array}$ & $\begin{array}{c}R_{1} \\
{[-]}\end{array}$ & $\begin{array}{c}N_{Q_{p}} \\
{[-]}\end{array}$ \\
\hline $1 / 3$ & 0.486 & 0.620 & 0.200 & 0.988 \\
\hline $1 / 4$ & 0.538 & 0.731 & 0.188 & 1.020 \\
\hline
\end{tabular}

Note: Data from ref. $[3,4]$ 
horizontal direction, the subregion of the liquid flowing horizontally along the bottom and, finally, the subregion of the liquid changing direction from the bottom (vertically) along the wall of the cylindrical vessel, when the volumetric flow rates of the liquid taking place in the downward and upward flows are the same.

\section{Acknowledgments}

The authors of this paper are grateful for financial support from the Czech Science Foundation (Grant No. 104/05/2500) and from the Czech Ministry of Education (Grant No. MSM6046137306).

\section{List of Symbols}

$b \quad$ width of baffle, $\mathrm{m}$

C impeller off-bottom clearance, $\mathrm{m}$

$D \quad$ impeller diameter, $\mathrm{m}$

$f_{\mathrm{ax}} \quad$ axial force, $\mathrm{N}$

$F_{\text {ax }} \quad$ dimensionless axial force

$H \quad$ height of water level, $\mathrm{m}$

$n \quad$ impeller speed, $\mathrm{s}^{-1}$ (rpm)

$N_{Q_{p}} \quad$ impeller flow rate number

$N_{Q_{b t}}$ dimensionless flow rate of the stream along the bottom

$p_{\text {ax }} \quad$ axial dynamic pressure, $\mathrm{Pa}$

$P_{\text {ax }} \quad$ dimensionless axial dynamic pressure

$Q_{p} \quad$ impeller pumping capacity, $\mathrm{m}^{3} \mathrm{~s}^{-1}$

$Q_{b t} \quad$ flow rate of the stream along the bottom, $\mathrm{m}^{3} \mathrm{~s}^{-1}$

$r \quad$ radial coordinate, $\mathrm{m}$

$R \quad$ dimensionless radial coordinate

$s_{p_{\mathrm{sx}}} \quad$ standard deviation of the axial dynamic pressure, $\mathrm{Pa}$

$S_{p_{\mathrm{sx}}} \quad$ dimensionless standard deviation of the axial $\mathrm{dy}$ namic pressure

$T$ diameter of mixing vessel, $\mathrm{m}$

$V \quad$ variance of the axial dynamic pressure

$W \quad$ width of impeller blade, $\mathrm{m}$

$\alpha \quad$ pitch angle, deg

$\rho \quad$ density of agitated liquid, $\mathrm{kg} \mathrm{m}^{-3}$

\section{Subscripts and superscripts}

1 related to the bottom subregion below the impeller rotor region

2 related to the bottom subregion at the vessel wall the mean time value

\section{References}

[1] Hrubý, M., Žaloudík, P.: Axial Component of Impellers (in Czech). Chemical Industry, Vol. 15 (1965), p. 469-472.

[2] Fořt, I., Tomeš, L.: Studies on Mixing. XVIII. The Action of a Stream from a Propeller Mixer on the Bottom of a Mixing Vessel. Collection of Czechoslovak Chemical Communications, Vol. 32 (1967), p. 3520-3529.
[3] Fořt, I., Eslamy, M., Košina, M.: Studies on Mixing. XXV. Axial Force of Axial Rotary Mixers. Collection of Czechoslovak Chemical Communications, Vol. 34 (1969), p. 3673-3691.

[4] Fořt, I.: Flow and Turbulence in Vessels with Axial Impellers. Chapter 14 of the Book Mixing, Theory and Practice, Vol. III (1986), New York: Academic Press, Inc., p. 133-197.

[5] Paglianti, A., Montante, G., Magelli, F.: Novel Experiments and Mechanistic Model for Macroinstabilities in Stirred Tanks, AICHE Journal (2006), Vol. 52, p. 426-436.

[6] Paglianti, A., Liu, Zh., Montante, G., Magelli, F.: Effect of Macroinstabilities in Single and Multiple Stirred Tanks, Industrial and Engineering Chemistry Research (in press).

[7] Kresta, S., Wood, P. E.: The Mean Flow Field Produced by a $45^{\circ} \mathrm{PBT}$. Changes of the Circulation Pattern due to Off-Bottom Clearance. Canadian Journal of Chemical Engineering, Vol. 71 (1993), p. 42-52.

Doc. Ing. Ivan Fořt, DrSc.

phone: +420224352713

fax: +420224310292

e-mail: Ivan.Fort@fs.cvut.cz

Department of Process Engineering

Czech Technical University in Prague

Faculty of Mechanical Engineering

Technická 4

16607 Praha 6, Czech Republic

Prof. Ing. Pavel Hasal, CSc.

phone: +420220443167

fax: +420220444320

e-mail: Pavel.Hasal@vscht.cz

Department of Chemical and Process Engineering

Prague Institute of Chemical Technology

Technická 5

16628 Praha 6, Czech Republic

Prof. Ing. Alessandro Paglianti

phone: +390512090403

fax: + 390516347788

e-mail: alessandro.paglianti@mail.ing.unibo.it

Prof. Ing. Franco Magelli

phone: + 390512090245

fax: + 390512090247

e-mail: franco.magelli@mail.ing.unibo.it

Department of Chemical, Mining and Environmental Engineering

University of Bologna

Via Terracini 28

40131 Bologna, Italy 